\title{
Connaissances, Attitudes et Pratiques des mères d'enfants sur l'allaitement exclusif
}

\section{Connaissances, Attitudes et Pratiques des mères d'enfants sur l'allaitement exclusif}

\author{
SIDIBE AK ${ }^{1}$, DIARRA AM ${ }^{1}$, DANIOKO Y², SANGARE $Y^{3}$, KONE K ${ }^{3}$ GUINDOU MT³ ${ }^{3}$ BELEM B ${ }^{3}$, \\ 1 Chargé de recherche au Centre de recherche, d'études et de documentation pour la survie de l'enfant, CRDOS, Bamako \\ 2 Médecin nutritionniste, CREDOS, Bamako \\ 3 Attaché de recherche au CRDOS, Bamako
}

\begin{abstract}
RESUME Introduction

L'Organisation Mondiale de la Santé recommande l'allaitement maternel exclusif jusqu'à six mois et conseille de le poursuivre partiellement jusqu'à deux ans en plus d'une alimentation de complément appropriée et sûre. L'objectif de notre travail était d'évaluer les connaissances, les attitudes et les pratiques des mères d'enfants de 0 à 59 mois sur l'allaitement maternel exclusif.

Méthode

L'étude de type transversal descriptif a été réalisée auprès d'enfants de 0 - 59 mois et leurs mères résidant à Bamako en commune $\mathrm{VI}$ depuis six mois de mai à juillet 2012.
\end{abstract}

\section{Résultats}

Parmi les mères enquêtées, $54,3 \%$ avaient l'âge compris entre 20-29 ans; l'âge moyen était de 26,86 ans et un écart type de 6,7 ans. Seulement $10 \%$ des mères ont pratiqué l'allaitement maternel exclusif jusqu'à six mois. La bonne santé de l'enfant a été la principale raison évoquée par les mères avec $52,3 \%$.

Selon $38,6 \%$ des enquêtées, l'allaitement maternel exclusif protège les enfants contre les maladies.

Les séances d'IEC au centre de santé pour $59 \%$ des enquêtées constituent la source d'information sur l'allaitement exclusif. Parmi les enquêtées $73,3 \%$ ont mis leur bébé au sein aussitôt après l'accouchement.

Avec une prévalence de $10 \%$ dans notre étude, l'AME n'est pas largement suivi par les mères d'enfants de 0-5 mois.

Mots clés : allaitement exclusif, connaissances, attitudes pratiques, mères, enfants.

\begin{abstract}
Introduction

The World Health Organization recommends exclusive breastfeeding for up to six months and advises that it be continued for up to two years in addition to appropriate and safe complementary feeding. The purpose of our work was to assess the knowledge, attitudes and practices of mothers of children aged 0 to 59 months on exclusive breastfeeding.
\end{abstract}

\section{Method}

The descriptive cross-sectional study was conducted from May 1 to July 1, 2012. The study population consisted of children aged $0-59$ months and mothers of children residing in commune $\mathrm{VI}$ for six months or more.

\section{Results}

Of the surveyed mothers, $54.3 \%$ were aged between 20 29 ; the average age was 26.86 years and a standard deviation of 6.7 years.

Only $10 \%$ of mothers had exclusive breastfeeding for up to six months. The good health of the child was the main reason mentioned by mothers with $52,3 \%$. According to $38.6 \%$ of the respondents, exclusive breastfeeding protects children against diseases. The IEC sessions at the health center for $59 \%$ of respondents are the source of information on exclusive breastfeeding.

Of the respondents, $73.3 \%$ breastfed immediately after giving birth. With a prevalence of $10 \%$ in our study, AME is not widely followed by mothers of 0-5 month's old children. Key words: Exclusive breastfeeding - knowledge attitudes - practices - mothers of children from 0 to 5 months.

\section{INTRODUCTION}

L'Organisation Mondiale de la Santé recommande l'allaitement maternel exclusif jusqu'à six mois et conseille de le poursuivre partiellement jusqu'à deux ans en plus d'une alimentation de complément appropriée et sûre. [1]

On estime à 2,7 millions le nombre annuel des décès d'enfants imputables à la sous-nutrition, soit $45 \%$ de tous les décès d'enfants. L'alimentation du nourrisson et du jeune enfant est un domaine primordial pour améliorer la survie des enfants et promouvoir une croissance et un développement sain. Les 2 premières années de vie d'un enfant sont particulièrement importantes car une nutrition optimale pendant cette période aura pour effet de réduire le taux de morbidité et de mortalité, ainsi que le risque de maladies chroniques, et de contribuer à un meilleur développement général. [2].

Le lait maternel est la principale source de nutriments pour l'enfant. Pendant les six premiers mois, l'allaitement maternel exclusif, c'est-à-dire sans aucun autre ajout, est recommandé par l'OMS parce qu'il transmet à l'enfant les anticorps de la mère et tous les éléments nutritifs nécessaires. En plus, le lait maternel, étant stérile, permet d'éviter la diarrhée et d'autres maladies [3]. 
En effet, l'OMS considère l'allaitement comme la meilleure manière de nourrir les jeunes enfants et de leur garantir la meilleure santé possible. Les institutions nationales et internationales de santé publique érigent donc des biopolitiques quand ils affirment, comme l'OMS, que « l'allaitement est le moyen idéal d'apporter aux nourrissons tous les nutriments dont ils ont besoin pour grandir et se développer en bonne santé. Le colostrum, sécrétion lactée jaunâtre et épaisse produite à la fin de la grossesse, constitue, ainsi que le préconise l'OMS, l'aliment parfait pour le nouveau-né qui doit commencer à s'alimenter dès la première heure qui suit la naissance [4].

L'allaitement maternel présente de nombreux avantages pour la santé de l'enfant et celle de sa mère. C'est la façon optimale de nourrir les nouveau-nés. En effet, l'allaitement maternel favorise la croissance physique et affective de l'enfant, privilégie le lien mère-enfant, renforce l'immunité de l'enfant et réduit les coûts liés à l'achat de lait artificiel et au recours aux soins [5-6]. L'allaitement optimal est tellement déterminant qu'il pourrait sauver chaque année la vie de 820000 enfants de moins de cinq ans [2].

L'allaitement exclusif au sein diminue la mortalité infantile imputable aux maladies courantes de l'enfance, comme les diarrhées ou les pneumonies, et il accélère la guérison en cas de maladie. II contribue à la santé et au bien-être des mères, aide à espacer les naissances, réduit le risque de cancer ovarien ou mammaire, augmente les ressources de la famille et du pays. C'est un moyen sûr et écologique d'alimenter l'enfant. Le lait maternel est donc sain, stérile, toujours à bonne température ; en plus de sa composition parfaite pour la santé, dont les effets se mesurent jusqu'à l'âge adulte, allaiter contribue également à consolider les liens mèreenfant [4].

Un allaitement maternel exclusif signifie qu'aucun autre aliment ni liquide n'est ajouté (sauf les médicaments) y compris l'eau, même en saison chaude, puisque le lait maternel est un aliment complet et répond aux besoins du nourrisson aussi bien en nutriments qu'en eau. Le lait maternel exprimé, c'est-à-dire provenant de la mère, d'une banque de lait ou d'une nourrice est considéré dans cette catégorie d'allaitement [7-8].

Dans le monde, de 2007 à 2014, on a constaté qu'en moyenne, seuls $36 \%$ environ des nourrissons de 0 à 6 mois sont exclusivement nourris au sein et $31 \%$ en Afrique subsaharienne [2].

En Afrique de l'ouest et du centre, seulement $22 \%$ des jeunes enfants de moins de 6 mois sont allaités exclusivement au sein [5].

Au Mali, dans le groupe d'âge de 0-5 ans, seulement un enfant sur trois soit 33\% n'a reçu que du lait maternel, c'est-à-dire qu'il a été exclusivement allaité au sein [3]. L'objectif de notre travail était d'évaluer les connaissances, les attitudes et les pratiques des mères d'enfant de 0 à 59 mois sur l'allaitement maternel exclusif

\author{
Materiel and Method \\ L'étude de type transversal descriptif a été réalisée du \\ 1er mai au 1er juillet 2012.
}

La population d'étude était constituée d'enfants de 0-59 mois et de toutes les mères d'enfants de 0 à 59 mois.

Toutes les mères d'enfants de 0 à 59 mois résidant en commune VI depuis six mois et plus, acceptant de répondre à nos questions étaient incluses dans l'étude.

N'ont pas été inclus dans cette étude toutes les mères d'enfants de 0- 59 mois résidant en commune $\mathrm{VI}$ depuis moins de 6 mois, absentes le jour de l'enquête et tout enfant dont l'âge était supérieur à 59 mois.

Nous avons utilisé la technique d'échantillonnage aléatoire en grappe à plusieurs degrés.

En prenant comme prévalence $(P)$ de l'AME chez les nourrissons de 0-59 mois $38 \%$ selon EDSM IV, en estimant la précision à $10 \%$ et en prenant en compte $10 \%$ de non répondants, nous avons obtenu une taille $n=$ 202.

Pour avoir le nombre de mères d'enfants de 0 à 59 mois par grappe nous avons divisé la taille par le nombre de grappe : $202 / 30=6,73 \approx 7$ mères d'enfants de 0 à 59 mois.

Dans chaque grappe retenue, 7 mères d'enfants de 0 à 59 mois ont été choisies au hasard. Si le quartier dispose plus d'une grappe, alors le nombre de mères d'enfants de 0 à 59 mois à enquêter sera 7 multiplié par le nombre de grappe que dispose ce quartier

Pour accéder aux concessions, nous nous sommes rendus chez le chef de quartier, nous avons jeté un crayon de papier et le bout du crayon a déterminé le chemin à suivre pour le choix de la première concession. Nous avons progressé ensuite de proche en proche.

Les variables mesurées étaient les caractéristiques sociodémographiques, les connaissances, les attitudes et les pratiques.

Les données ont été saisies à l'aide du logiciel épi DATA version 3.1 et analysées sur SPSS version 18.0.

Le test de chi carré de Pearson a été utilisé avec un seuil de 0,05 pour rechercher la liaison entre les variables dépendantes et les variables indépendantes. La relation a été considérée comme significative pour $p<0,05$.

Pour des considérations éthiques, des lettres ont été adressées aux autorités sanitaires pour l'information et leur accord avant le démarrage de la collecte des données.

Des fiches de consentement éclairé ont été administrées aux enquêtées avant le démarrage des travaux.

RESULTATS

Caractéristiques socio démographique des enquêtées :

- Ages et situation matrimoniale

Dans notre échantillon, $54,3 \%$ des mères avaient l'âge compris entre 20-29 ans. L'âge minimal est de 15 ans et l'âge maximal de 49 ans. Parmi ces mères enquêtées, $77,1 \%$ étaient mariées. 


\section{- Profession et niveau d'instruction}

Les ménagères représentaient $54,8 \%$, toutes étaient des mères paucipares (Tableau I) et presque $42 \%$ n'étaient pas scolarisées.

- Pratique de l'allaitement exclusif jusqu'à 6 mois Sur les 210 mères enquêtées, seules 21 ont pratiqué l'allaitement maternel exclusif jusqu'à six mois soit $10 \%$.

\section{Connaissance des mères sur l'allaitement exclusif}

- des avantages

Dans notre travail, moins de la moitié des mères enquêtées ont cité la protection contre les maladies $(38,6 \%)$ suivie de la croissance de l'enfant $(19,5)$ comme des avantages de l'allaitement exclusif.

- de l'âge de la diversification alimentaire et de l'ablactation

La majorité des enquêtées soit $90 \%$ pensent qu'il faut diversifier l'alimentation de l'enfant après six mois.

- de l'âge de l'ablactation

Dans notre étude $73,3 \%$ des enquêtées pensent qu'il faut arrêter l'allaitement maternel entre 12-24 mois.

- Obstacles à l'allaitement maternel

Nous avons trouvé $27,9 \%$ de mères qui ont rencontré des obstacles au cours de l'allaitement. La douleur des seins avec 38,5\%, suivie de l'insuffisance de sécrétion lactée $(31,5 \%)$ sont les obstacles auxquels les mères ont été confrontées.

\section{Informations sur l'allaitement maternel exclusif}

- Source d'information pendant les CPN

$\mathrm{Au}$ cours des consultations prénatales, la source d'information la plus citée est le centre de santé $(69,5)$ suivie des médias (23\%)

- Source d'information pendant l'accouchement Les résultats de notre travail ont montré que moins de la moitié des mères $(43,8)$ ont reçu des informations sur l'allaitement maternel exclusif pendant l'accouchement.

- Source d'information après l'accouchement Les sages-femmes ont été citées par les mères $(78,3 \%)$ comme principale source d'information suivies des médecins $(20,7 \%)$

Attitudes et pratiques des femmes sur l'allaitement exclusif

- Délai de mise au sein après l'accouchement Dans notre étude, $73,3 \%$ des mères ont mis leur bébé au sein aussitôt après accouchement (Tableau II). Pour $67,6 \%$ des mères enquêtées, les bébés ont été allaités à leur demande.

\section{- Administration du colostrum}

Dans notre étude, $97,6 \%$ des femmes ont affirmé avoir donné le colostrum à l'enfant et $52,3 \%$ d'elles ont évoqué la bonne santé de l'enfant comme principale raison de cette pratique.

- Aliments donnés en plus du sein

L'aliment le plus donné à l'enfant était l'eau et la tisane

$(38,1 \%)$, suivi de l'eau et du lait artificiel $(24,9 \%)$.
Les facteurs qui influencent la pratique de l'allaitement maternel exclusif

Dans notre étude, nous n'avons mis en évidence aucune relation avec les variables :'âge, parité, statut matrimonial, profession, niveau d'instruction et la pratique de l'allaitement maternel exclusif (Tableau III).

\section{Discussion}

Cette enquête visait à étudier les connaissances, les attitudes et les pratiques des mères sur l'allaitement maternel exclusif.

\section{Caractéristiques socio démographique des} enquêtées :

- Ages et situation matrimoniale

Nos résultats ont montré que plus de la moitié des enquêtées $(54,3 \%)$ avaient un âge compris entre 20-29 ans avec une moyenne de 26,86 ans et un écart type de 6,7 ans. Ce résultat est très proche de ceux trouvés par Amed Coulibaly et al, 26,56 ans $\pm 5,05$ ans [9] et Djadou $\mathrm{KE}$ et al, 27,5 ans [10]

Parmi nos mères enquêtées, une majorité $(77,1 \%)$ étaient mariées. Ce résultat est supérieur à celui trouvé par d'Amed Coulibaly et al avec 26,60\% [9]. Par contre, la proportion de mères mariées dans notre étude est inférieure à celle trouvée par Adedemy $\mathrm{J} D$ et al. qui était de $94,1 \%$ [11].

\section{- Profession et niveau d'instruction}

Dans notre étude, les ménagères étaient les plus représentées soit $54,8 \%$ et moins de la moitié $(41,9 \%)$ étaient non scolarisées (Tableau I). Ces résultats sont supérieurs à celui d'Amed Coulibaly et al. qui est de $12,23 \%$ de ménagère et $19,89 \%$ de non scolarisé [9] et également à celui trouvé par ADEDEMYJ $D$ et al où les ménagères étaient les plus représentées avec une proportion de $30,6 \%$ et $23,5 \%$ de non scolarisées [11].

- Pratique de l'allaitement exclusif jusqu'à 6 mois

Au cours de notre étude, nous n'avons trouvé que $10 \%$ de mère qui pratiquaient l'AME jusqu'à six mois. $\mathrm{Ce}$ qui reste faible, comparée aux taux trouvés par Amed Coulibaly et al, 16,67\% [9] Demmouche et al, 32\% [7] au niveau national $33 \%$ [3],Toni $L$ et al $30.67 \%$ [12 ] et DIARRA et al., 36,5\% [13].

La principale raison évoquée par ces mères $(52,3 \%)$ qui pratiquaient de l'AME jusqu'à 6 mois, est la bonne santé de l'enfant suivie du renforcement des relations mèresenfant $(19,1)$.Ce même argument, la santé du bébé, est ressorti dans les résultats de Demmouche et al. où $32,35 \%$ des mères l'ont citée comme la raison de la pratique de l'AME [7].

\section{Connaissance des mères sur l'allaitement exclusif - des avantages}

Nous avons trouvé dans nos résultats que moins de la moitié des mères $(38,6 \%)$ ont cité la protection contre les maladies majoritaires, suivie de la croissance de l'enfant $(19,5)$ comme avantage de l'allaitement maternel exclusif Ce résultat est nettement différent de celui trouvé 
par Folly MESSAN et al. où $55,71 \%$ des nourrices enquêtées ne connaissaient pas les avantages de l'allaitement maternel exclusif contre $03,57 \%$ qui ont cité la protection de la mère contre les maladies comme avantage de l'allaitement maternel exclusif [14].

- de l'âge de la diversification alimentaire et de l'ablactation

La majorité de nos enquêtées $(90 \%)$ pensent qu'il faut diversifier l'alimentation de l'enfant après les six premiers mois. Ce qui est conforme aux recommandations de l'OMS en matière de l'âge de diversification alimentaire ou âge d'introduction d'aliments de complément [2].

- de l'âge de l'ablactation

De l'avis de $73,3 \%$ de nos enquêtées l'âge du sevrage de l'enfant se situe entre 12-24 mois. Ce qui est très différents du résultat de Mahamat Bechir au Tchad où l'âge du sevrage intervient plus tôt (avant un an) pour les enfants sédentaires au contraire de ceux nomades où il intervient à plus d'un an[ 15 ].

\section{- Obstacles à l'allaitement materne}

Nous avons trouvé $27,9 \%$ de mères qui ont rencontré des obstacles au cours de l'allaitement. La douleur des seins avec 38,5\%, suivie de l'insuffisance de sécrétion lactée $(31,5 \%)$ sont les obstacles auxquels les mères ont été confrontées. Par ailleurs, Djadou KE et al. ont trouvé dans leur étude au Togo que la pression de l'entourage $(34,6 \%)$ et le manque de lait $(27,9 \%)$ étaient les obstacles à l'AME [10]

\section{Informations sur l'allaitement exclusif}

- Source d'information pendant les CPN

Au cours des consultations prénatales, la source d'information la plus citée est le centre de santé $(69,5)$ suivie des médias (23\%). Ces résultats corroborent avec ceux trouvés par Laure SAGE dans son mémoire pour le diplôme de sage-femme d'état en 2014 , où $63,06 \%$ des patientes avaient suivi des séances de préparation à la naissance et avaient obtenu à $57,65 \%$ une information sur l'allaitement à cette occasion. Les sources médiatiques (Internet et télévision) ne représentaient finalement que $25,23 \%$ [16].

\section{- Source d'information après l'accouchement}

Les sages-femmes ont été citées par les mères (78,3\%) comme étant la principale source d'information suivies des médecins $(20,7 \%)$. Ce qui concorde avec les résultats trouvés dans l'étude de N.R.Diagne-Guèye et al. au Sénégal où $59 \%$ des mères avaient sollicité des professionnels de santé comme source d'information [17].

Attitudes et pratiques des femmes sur l'allaitement exclusif

\section{- Délai de mise au sein après l'accouchement}

Dans notre étude, aussitôt après l'accouchement 73,3\% de nos mères ont mis au sein leur bébé. (Tableau II). Ce résultat est nettement supérieur à celui trouvé par DIARRA et al dans leur étude avec un taux $42,4 \%$ de mise au sein précoce [14] et également supérieur à celui trouvé par N.R. Diagne-Guèye et al. au Sénégal où $48 \%$ des mères ont mis leur enfant au sein dans l'heure qui a suivi la naissance [17]

\section{- Administration du colostrum}

Nos résultats ont montré que $97,6 \%$ des femmes enquêtées ont donné le colostrum à leur bébé et la principale raison de cette pratique pour plus de la moitié $(52,3 \%)$ de ces mères est la bonne santé de l'enfant. Ce résultat corrobore avec celui de N.R. Diagne-Guèye et al. où toutes les 44 mères déclarent n'avoir pas jeté le ${ }^{2}<<<$ colostrum [17].

\section{- Aliments donnés en plus du sein}

Dans notre travail, « eau et tisane » $(38 \%)$, suivi de « eau et lait artificiel $»(24,9 \%)$ étaient les aliments les plus donnés à l'enfant. Dans l'étude de N.R. Diagne-Guèye et al.au Sénégal, $63 \%$ des mères avaient donné du lait et $36 \%$ d'entre elles avaient donné en plus du lait maternel le « Tokental » [17].

Facteurs qui influencent la pratique de l'allaitement exclusif

Notre étude n'a mis en évidence aucune relation qui influence la pratique de l'allaitement exclusif (Tableau III).

\section{Conclusion}

La prévalence de l'allaitement maternel dans notre étude était faible par rapport au niveau national. Pour améliorer cette prévalence de l'allaitement exclusif jusqu'à l'âge de six mois dans la commune VI du district de Bamako, il est nécessaire d'améliorer le niveau d'instruction des femmes pour une réception plus aisée et facilitée des conseils donnés par le personnel de santé. II est de la responsabilité des professionnels de santé de les informer des bienfaits de la pratique de l'allaitement maternel exclusif.

\section{REFERENCES BIBLIOGRAPHIQUES}

1. Moussa Salatou Diagana, tHélène Kane. Alimentation des nourrissons à Nouakchott : entre recommandations médicales et instructions des grand-mères, Santé Publique 2016; 28 (2): $p$ 235-243

2. Organisation Mondiale de la Santé, Centre des médias, Alimentation du nourrisson et du jeune enfant, fév 2018

3. Cellule de Planification et de Statistique (CPS/SSDSPF), Institut National de la Statistique (INSTAT/MPATP), INFO-STAT et ICF International, 2014.Enquête Démographique et de Santé au Mali 2012-2013. Rockville, Maryland, USA : CPS, INSTAT, INFO-STAT et ICF International.

4. Nathalie Gr. « Allaiter sur le web ». Entre biopouvoir et rituels numériques, Les Cahiers du numérique, 2013 ; 9(3) : p 63-81

http://www.cairn.info/revue-les-cahiers-dunumerique-2013-3-page-63.htm.

5. Traoré $\mathrm{M}$ et al. « Facteurs associés à l'allaitement maternel exclusif chez les mères d'enfants de 24 
mois à Bamako », Santé Publique $2014 ; 26$ (2) : p 259-265.

6. Organisation Mondiale de la Santé, Daniel Epstein, Responsable de Communication, Genève. Allaitement maternel: Communiqué de presse 30 juil. 2013.

7. Demmouche A., Beddek F, Moulessehoul S. Les déterminants du choix de l'allaitement chez une population de l'ouest de l'Algérie (ville de Sidi Bel Abbes). Antropo, ISSN-e 1578-2603, 2013 ; 30 : 6169

8. Rigourd V, Aubry S, Tasseau A et al. Allaitement maternel : bénéfices pour la santé de l'enfant et de sa mère. $J$ de pédiatr et puéricult, avr, $2013 ; 26: p$ 90-99

9. Coulibaly A et al. « Facteurs socioprofessionnels et pratique de l'allaitement exclusif par les primipares à Abidjan (Côte d'Ivoire) », Santé Publique 2014; 26(4) : p 555-562.

10. Djadou KE, Agbeko F, Guédéhoussou T, Dizewé K, Azoumah $\mathrm{K}$, Agbèrè AD. Evaluation de l'allaitement maternel exclusif chez les enfants de 0 à six mois dans le district de Tchaoudjo (Togo), J Afr Pediatr Genet Med 2018; (4) : p 30-36

11. Adedemy J D, Bagnan-Tossa L , Noudamadjo A, Agossou $\mathrm{J}$ et Hounhakou P. Fréquence et facteurs associés à la pratique de l'allaitement maternel exclusif de 0 a 6 mois à l'hôpital de la mère et de l'enfant LAGUNE (HOMEL) de Cotonou, Journal de la Société de Biologie Clinique du Bénin, 2014 ; ( $021)$ : p 38-44.
12. Lubala K T, Mulangu $M A$, Nyenga $M A$ et al. Allaitement maternel et diversification alimentaire à Lubumbashi (République Démocratique du Congo): besoin urgent d'éducation des mères pour le changement des habitudes Pan African Medical Journal. 2013; 14: 142.

13. Diarra I, Yarro F. Prévalence et facteurs associés à l'allaitement exclusif chez les enfants de 0 à 6 mois dans la ville de Ouidah au Benin en 2009, Mali Santé Publique 2013; 3 (001)

14. DAKPO C P., MESSAN F, NAKOU A S, Dénis A. LODONOU R. Stéphinx. M. Contribution de l'allaitement maternel exclusif à la santé communautaire en Afrique subsaharienne : cas de la commune de Dangbo au Bénin. Université d'Abomey-Calavi - Bénin, janv. 2013c;p 231-254. https://www.researchgate.net/publication/273319958 [en ligne] consultée le 02 septembre 2018

15. Bechir $M$ \& Tidjani $A$, coordinateurs, Revue scientifique du Tchad. Les Actes Scientifiques du Forum National sur la Nutrition et l'Alimentation au Tchad, organisé du 28 au 30 avril 2015.

16. Sage L. Connaissances sur l'allaitement maternel des femmes allaitantes avant leur sortie de maternité, [mémoire pour le diplôme d'état de sagefemme]. Chu Estaing de Clermont-Ferrand, 2014.

17. Diagne- N.R Guèye, Diack $A$ Mbaye Dramé $M$ et al. Connaissances et pratiques de mères sénégalaises sur l'allaitement au sein, Journal de pédiatrie et de puériculture $2011 ; 24$ : p 161-166. 
Tableau I : Répartition des mères d'enfants de 0 à 59 mois selon les caractéristiques sociodémographiques et la parité

\begin{tabular}{|c|c|c|}
\hline Caractéristiques [n= 210] & Effect & $\%$ \\
\hline \multicolumn{3}{|l|}{ Tranche d'âge } \\
\hline $15-19$ ans & 30 & 14,3 \\
\hline $20-29$ ans & 114 & 54,3 \\
\hline $30-39$ ans & 55 & 26,2 \\
\hline 40- 49 ans & 11 & 5,2 \\
\hline \multicolumn{3}{|l|}{ Statut matrimonial } \\
\hline Mariée & 162 & 77,1 \\
\hline Célibataire & 40 & 19,1 \\
\hline Divorcée & 4 & 1,9 \\
\hline Veuve & 4 & 1,9 \\
\hline \multicolumn{3}{|l|}{ Niveau d'instruction } \\
\hline Non scolarisée & 88 & 41,9 \\
\hline Primaire & 60 & 28,6 \\
\hline Secondaire & 30 & 14,3 \\
\hline Supérieur & 32 & 15,2 \\
\hline \multicolumn{3}{|l|}{ Profession } \\
\hline Ménagère & 115 & 54,8 \\
\hline Elève /Etudiante & 44 & 21,0 \\
\hline Commerçante & 25 & 11,9 \\
\hline Fonctionnaire & 20 & 9,5 \\
\hline Autres & 6 & 2,8 \\
\hline \multicolumn{3}{|l|}{ Parité } \\
\hline Primipare & 70 & 33,3 \\
\hline Paucipare & 115 & 54,8 \\
\hline Multipare & 21 & 10,0 \\
\hline Grande multipare & 4 & 1,9 \\
\hline
\end{tabular}

Tableau II : Répartition des mères d'enfants de 0 à 59 mois enquêtées selon leurs raisons de la pratique de l'allaitement exclusif et la source d'information de leurs aptitudes et pratiques

\begin{tabular}{lcc}
\hline Variables Caractéristiques [n= 210] & $\mathbf{n}$ & $\%$ \\
\hline Raisons & 11 & \\
Bonne santé de l'enfant & 4 & 19,3 \\
Renforce relation mère-enfant & 3 & 14,3 \\
Sur avis du médecin & 2 & 9,5 \\
Contraception & 1 & 4,8 \\
Economique & & \\
Sources d'information & 124 & $\mathbf{5 9 , 0}$ \\
IEC au centre de santé & 50 & 23,8 \\
A travers les medias & 22 & 10,5 \\
Agent de santé & 14 & 6,7 \\
Amies & & \\
Délai de mise au sein & 154 & 73,3 \\
Aussitôt après accouchement & 49 & 23,3 \\
Entre 1ère ère et 8èmeème heure & 7 & 3,4 \\
Plus de 8 heures & & \\
Fréquence des tétées/jour & 142 & 67,6 \\
A la demande & 44 & 21,0 \\
Moins de 10 fois & 24 & 11,4 \\
Plus de 10 fois & & \\
Administration du colostrum & $\mathbf{2 0 5}$ & $\mathbf{9 7 , 6}$ \\
Oui & 5 & 2,4 \\
Non & & \\
Raisons de l'administration du colostrum & 106 & $\mathbf{5 2 , 3}$ \\
Bon pour la santé de l'enfant & 51 & 24,3 \\
Ne sait pas & 48 & 23,4 \\
Par conseil de l'agent de santé & & \\
\hline &
\end{tabular}

Tableau III : Répartition des mères d'enfants de 0 à 59 mois enquêtées selon la pratique de l'AME en fonction des caractéristiques sociodémographiques et de l'information à l'accouchement

Caractéristiques [ $n=210]$

\begin{tabular}{cccc}
\hline & \multicolumn{3}{c}{ Allaitement exclusif } \\
& Oui & & Non \\
& $\%$ & $\mathbf{n}$ & $\%$
\end{tabular}

\begin{tabular}{|c|c|c|c|c|c|}
\hline Age & & & & & 0,84 \\
\hline$<30$ ans & 14 & 6,7 & 130 & 61,9 & \\
\hline$\geq 30$ ans & 7 & 3,3 & 59 & 28,1 & \\
\hline Parité & & & & & 0,62 \\
\hline Multipare & 13 & 6,2 & 127 & 60,5 & \\
\hline Primipare & 8 & 3,8 & 62 & 25,5 & \\
\hline Statut Matrimonial & & & & & 0,98 \\
\hline Celibataire & 4 & 1,9 & 36 & 17,1 & \\
\hline Mariée & 16 & 7,6 & 146 & 69,5 & \\
\hline Profession & & & & & 1,14 \\
\hline Ménagère & 10 & 4,8 & 105 & 50,0 & \\
\hline Etudiante/Elève & 7 & 3,3 & 37 & 17,6 & \\
\hline Commerçant & 1 & 0,5 & 24 & 11,4 & \\
\hline Fonctionnaire & 0 & 0,0 & 13 & 6,2 & \\
\hline Autres* & 3 & 1,4 & 10 & 4,8 & \\
\hline Niveau d'Instruction & & & & & 0,92 \\
\hline Scolarisée & 12 & 5,7 & 110 & 52,4 & \\
\hline Non scolarisée & 9 & 4,3 & 79 & 37,6 & \\
\hline Information/accouchement & & & & & 0,19 \\
\hline Oui & 12 & 5,7 & 80 & 38,1 & \\
\hline Non & 9 & 4,3 & 109 & 51,9 & \\
\hline Delai de mise au sein & & & & & 0,62 \\
\hline Scolarisée & 91 & 43,3 & 31 & 14,8 & \\
\hline Non scolarisée & 63 & 30,0 & 25 & 11,9 & \\
\hline
\end{tabular}

